\title{
Liter per Hour per Microgram per Kilogram per Day
}

National Cancer Institute

\section{Source}

National Cancer Institute. Liter per Hour per Microgram per Kilogram per Day. NCI

Thesaurus. Code C120747.

A unit of concentration equal to liter per hour divided by microgram per kilog ram per day. 